\title{
Topic Introduction
}

\section{Genome Editing in Human Pluripotent Stem Cells}

\author{
Cory Smith, ${ }^{1,2,3}$ Zhaohui Ye, ${ }^{1,2}$ and Linzhao Cheng ${ }^{1,2,3,4}$ \\ ${ }^{1}$ Division of Hematology, Department of Medicine, Johns Hopkins University School of Medicine, Baltimore, \\ Maryland 21205; ${ }^{2}$ Stem Cell Program, Institute for Cell Engineering, Johns Hopkins University School of \\ Medicine, Baltimore, Maryland 21205; ${ }^{3}$ Predoctoral Training Program in Human Genetics, Johns Hopkins \\ University School of Medicine, Baltimore, Maryland 21205
}

Pluripotent stem cells (PSCs), defined by their capacity for self-renewal and differentiation into all cell types, are an integral tool for basic biological research and disease modeling. However, full use of PSCs for research and regenerative medicine requires the ability to precisely edit their DNA to correct disease-causing mutations and for functional analysis of genetic variations. Recent advances in DNA editing of human stem cells (including PSCs) have benefited from the use of designer nucleases capable of making double-strand breaks (DSBs) at specific sequences that stimulate endogenous DNA repair. The clustered, regularly interspaced short palindromic repeats (CRISPR)-Cas9 system has become the preferred designer nuclease for genome editing in human PSCs and other cell types. Here we describe the principles for designing a single guide RNA to uniquely target a gene of interest and describe strategies for disrupting, inserting, or replacing a specific DNA sequence in human PSCs. The improvements in efficiency and ease provided by these techniques allow individuals to precisely engineer PSCs in a way previously limited to large institutes and core facilities.

Embryonic stem cells (ESCs) are derived from the inner cell mass of a developing blastocyst and can be expanded in vitro for decades without losing their full potential for differentiation and self-renewal. ESCs hold great potential for basic biological research, disease modeling, and as a cell source for regenerative medicine. However, because of the destructive nature of their derivation, an autologous source of ESCs is not available without therapeutic cloning. Also, the high monetary and ethical costs associated with obtaining oocytes limit the practical applications and scalability of their use. The discovery that four simple transcription factors could reprogram somatic fibroblasts to an embryonic-like state (i.e., induced PSCs) provides an autologous cell source for individualized regenerative medicine and basic research, including disease modeling, high-throughput drug screening, and access to human cell types previously unavailable for live study, including neurons and astrocytes. The full realization of these PSCs will rely on the ability to precisely manipulate their DNA to investigate the effects of these variants in isolation or to cure a disease-causing variant ex vivo before cell-based regenerative therapy.

Gene targeting using a double-stranded DNA donor with long homology arms (1-4 kb) is a wellestablished technique across many animal models and cell lines. Although this approach has been used in mouse PSCs, the many technical limitations using human PSCs prevented successful modification of this technique: Single cells rarely survive without cell-to-cell contacts and signals that aid in the formation of the early colony after the stress of electroporation, and the rates of homologous recom-

\footnotetext{
${ }^{4}$ Correspondence: Icheng2@jhmi.edu

(C) 2016 Cold Spring Harbor Laboratory Press

Cite this introduction as Cold Spring Harb Protoc; doi:10.1101/pdb.top086819
} 
bination are significantly lower in human PSCs than in mice. The first limitation was addressed by the development of Rho-associated kinase inhibitor that dramatically increases survival and proper colony formation after the single-cell digestion of human PSCs required for electroporation; the second issue was addressed by the development of sequence-specific nucleases that generate a DSB in DNA at a targeted location, increasing the efficiency of recombination at that site by several orders of magnitude (Hockemeyer et al. 2009; Zou et al. 2009). DSBs are detected by the cell's endogenous DNA repair machinery, which proceeds through either the error-prone nonhomologous end joining (NHEJ) pathway that often results in small indel mutations around the break site or by homologydependent repair (HDR) using a DNA donor template. These tools allow researchers to both disrupt genes through NHEJ and to create single-nucleotide variants with an HDR donor to determine its effects in isolation when compared with an otherwise isogenic cell line.

\section{CRISPR-Cas EDITING OF PSCs}

The Streptococcus pyogenes-derived adaptive bacterial immune system CRISPR-Cas degrades foreign DNA in a sequence-specific manner guided by short guide RNAs (gRNAs) to induce a DSB. In particular, the type II CRISPR system only requires a single protein component, Cas9, and a single gRNA to determine its target and cleave DNA. This system has been successfully adapted for mammalian expression (Cong et al. 2013; Mali et al. 2013). A major advantage of CRISPR-Cas9 is the ability to rapidly design, synthesize, assemble, and test gRNAs targeting new genes or noncoding regions of interest, as well as the ability to multiplex by transfecting additional gRNAs. Several studies-largely conducted using cancer cell lines (e.g., HEK 293T or U20S)—revealed higher than expected off-target mutagenesis, including some sites that were altered more frequently than the intended target and other sites that included up to five mismatches compared with the gRNA (Fu et al. 2013; Hsu et al. 2013; Mali et al. 2013); further screening in animal embryos and human stem cells reported minimal off-target effects (Wang et al. 2013; Niu et al. 2014; Wu et al. 2014). Several complementary approaches to screen human PSCs for potential off-target mutagenesis such as targeted sequencing of top sites predicted most likely to cleave based on sequence similarity revealed high specificity of these gRNAs tested in human PSCs relative to HEK 293Ts (Mali et al. 2013; Smith et al. 2014; Li et al. 2015). Additionally, whole-genome sequencing conducted on CRISPRCas9-targeted PSCs revealed new mutations in each clone, but none were similar to the gRNA target site or recurrent between the clones, indicating that genome editing of PSCs can be performed with minimal off-target mutagenesis genome-wide (Smith et al. 2014; Suzuki et al. 2014; Veres et al. 2014; Yang et al. 2014).

There are many approaches for genome editing to either knock out a gene, induce a specific single-nucleotide change, or insert a larger fragment for transgene expression or to add a fluorescent tag (Fig. 1). The most basic strategy involves a single guide RNA targeting the protein coding sequence of a gene to disrupt expression by a frameshift mutation, often leading to degradation through nonsense-mediated decay. If two gRNAs are used in close proximity $(\sim 20 \mathrm{bp}-10 \mathrm{~kb})$ the intervening sequences is deleted, often with precise breakpoints 3 nucleotides upstream of the NGG, although small indels are observed around the junction at a low frequency. This technique can be used to delete exons that could not otherwise be targeted because of highly conserved domains and also provides a tool to excise defined regions, making it a promising approach to investigate noncoding variants such as regulatory elements over a broad range of deletion sizes. To induce specific mutations (e.g., singlenucleotide changes), the classic approach uses a double-stranded DNA donor plasmid with homology arms of $\sim 0.5-2 \mathrm{~kb}$. Using CRISPR-Cas9, this approach can produce dramatically higher efficiencies $(\sim 10 \%)$ without using drug selection (Byrne et al. 2015) and can also be used to insert transgenes into a predetermined safe harbor locus (e.g., the adeno-associated virus integration site 1) or to tag a lineage-specific gene to monitor expression during differentiation. Another targeting approach uses a single-stranded DNA oligonucleotide (ssODN) of $\sim 70-110$ bases, although 90 bp was optimal in 
C. Smith et al.

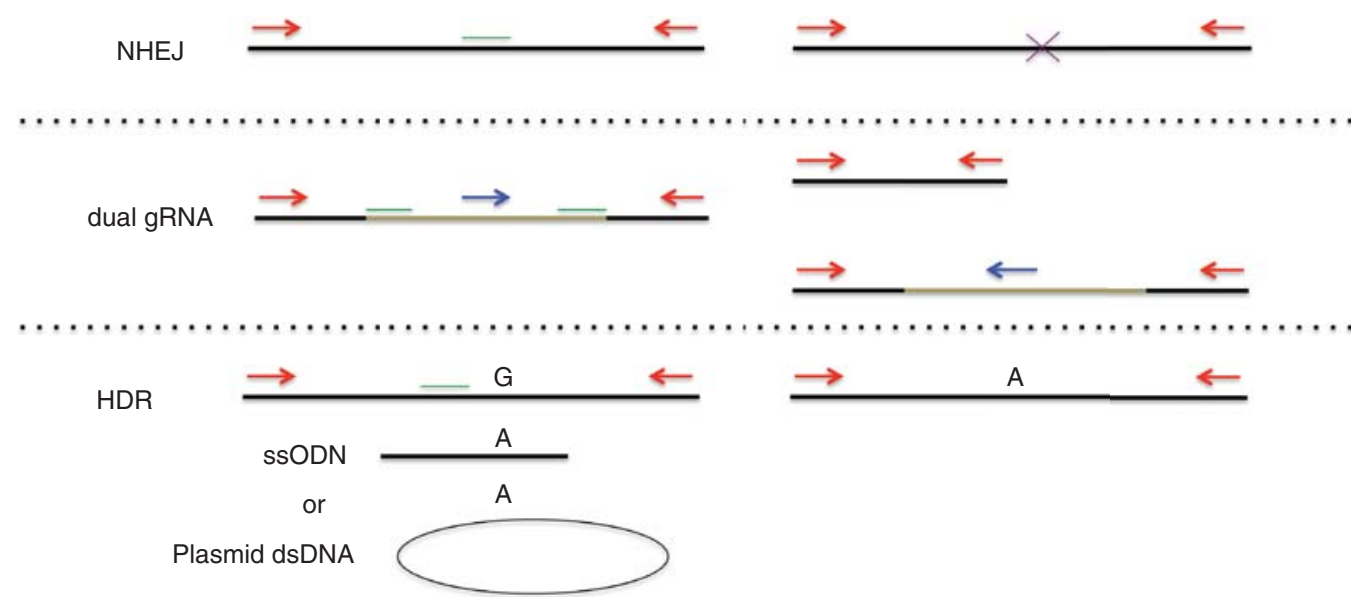

FIGURE 1. Targeting approaches for genome editing. NHEJ uses a single guide RNA (green) targeting the protein coding sequence of a gene to disrupt expression by a frameshift mutation $(\times)$. Dual guide RNA uses two gRNAs in close proximity to delete the intervening sequences (blue arrow). HDR can be used with either a single-stranded DNA oligonucleotide (ssODN) or a double-stranded DNA donor (plasmid dsDNA) to induce specific mutations.

human PSCs (Yang et al. 2013). The same study also reported that it was ideal to use the strand complementary to the gRNA for the ssODN as opposed to the same strand. It is best to design the gRNA as close to the mutation as possible (ideally, within 10 nucleotides for ssODNs or 200 nucleotides for plasmid donors) (Xie et al. 2014).

In Protocol: Protocol for Genome Editing in Human Pluripotent Stem Cells (Smith et al. 2016), we describe a method for genome editing in PSCs with CRISPR-Cas9 to disrupt or delete a predetermined DNA sequence or to alter a nucleotide of interest for further study. Targeting considerations are discussed to design gRNAs for both knockout and knock-in strategies that have the least chance of off-target mutagenesis predicted bioinformatically and tested experimentally in the PSC line of interest. We also provide a simplified procedure for gRNA synthesis and assembly and discuss considerations for transfection-quality DNA needed to modify the traditionally difficult-to-transfect PSCs. Briefly, transfection is achieved through electroporation using the Nucleofector 4D (Lonza), although similar efficiencies in PSCs can be obtained using other technologies (e.g., Life Technologies' Neon Transfection System). With initially 1\% NHEJ disruption efficiencies for Cas9 in human PSCs (Mali et al. 2013) clonal isolation and screening for targeted clones remains the most time-consuming and costly part of the procedure as hundreds to thousands of clones need to be screened to find several with the intended mutation. Although feasible, several improvements have been made to increase the efficiency of editing by using either fluorescence-assisted cell sorting for Cas9_GFP ${ }^{+}$cells (Ding et al. 2013) or by integrating a dox-inducible Cas9 system (González et al. 2014). The future application of these genome-editing techniques to PSCs will allow cell-based functional investigations of any genetic variant of interest such as the incoming torrent of genome-wide association data or potential causative variants in rare case anomalies at the extremes of phenotypes.

\section{REFERENCES}

Byrne SM, Ortiz L, Mali P, Aach J, Church GM. 2015. Multi-kilobase homozygous targeted gene replacement in human induced pluripotent stem cells. Nucleic Acids Res 43: e21.

Cong L, Ran FA, Cox D, Lin S, Barretto R, Habib N, Hsu PD, Wu X, Jiang W, Marraffini LA, et al. 2013. Multiplex genome engineering using CRISPR/Cas systems. Science 339: 819-823.

Ding Q, Regan SN, Xia Y, Oostrom LA, Cowan CA, Musunuru K. 2013. Enhanced efficiency of human pluripotent stem cell genome editing through replacing TALENs with CRISPRs. Cell Stem Cell 12: 393-394.
Fu Y, Foden JA, Khayter C, Maeder ML, Reyon D, Joung JK, Sander JD. 2013. High-frequency off-target mutagenesis induced by CRISPR-Cas nucleases in human cells. Nat Biotechnol 31: 822-826.

González F, Zhu Z, Shi ZD, Lelli K, Verma N, Li QV, Huangfu D. 2014. An iCRISPR platform for rapid, multiplexable, and inducible genome editing in human pluripotent stem cells. Cell Stem Cell 15: 215226.

Hockemeyer D, Soldner F, Beard C, Gao Q, Mitalipova M, DeKelver RC, Katibah GE, Amora R, Boydston EA, Zeitler B, et al. 2009. Efficient 
targeting of expressed and silent genes in human ESCs and PPSCs using zinc-finger nucleases. Nat Biotechnol 27: 851-857.

Hsu PD, Scott DA, Weinstein JA, Ran FA, Konermann S, Agarwala V, Li Y, Fine EJ, Wu X, Shalem O, et al. 2013. DNA targeting specificity of RNAguided Cas9 nucleases. Nat Biotechnol 31: 827-832.

Li HL, Fujimoto N, Sasakawa N, Shirai S, Ohkame T, Sakuma T, Tanaka M, Amano N, Watanabe A, Sakurai H, et al. 2015. Precise correction of the dystrophin gene in Duchenne muscular dystrophy patient induced pluripotent stem cells by TALEN and CRISPR-Cas9. Stem Cell Rep 4: 143-154.

Mali P, Yang L, Esvelt KM, Aach J, Guell M, DiCarlo JE, Norville JE, Church GM. 2013. RNA-guided human genome engineering via Cas9. Science 339: 823-826.

Niu Y, Shen B, Cui Y, Chen Y, Wang J, Wang L, Kang Y, Zhao X, Si W, Li W, et al. 2014. Generation of gene-modified cynomolgus monkey via Cas9/ RNA-mediated gene targeting in one-cell embryos. Cell 156: 836-843.

Smith C, Gore A, Yan W, Abalde-Atristain L, Li Z, He C, Wang Y, Brodsky RA, Zhang K, Cheng L, et al. 2014. Whole-genome sequencing analysis reveals high specificity of CRISPR/Cas9 and TALEN-based genome editing in human iPSCs. Cell Stem Cell 15: 12-13.

Smith C, Ye Z, Cheng L. 2016. Protocol for genome editing in human pluripotent stem cells. Cold Spring Harb Protoc doi: 10.1101/pdb .prot090217.

Suzuki K, Yu C, Qu J, Li M, Yao X, Yuan T, Goebl A, Tang S, Ren R, Aizawa E, et al. 2014. Targeted gene correction minimally impacts wholegenome mutational load in human-disease-specific induced pluripotent stem cell clones. Cell Stem Cell 15: 31-36.
Veres A, Gosis BS, Ding Q, Collins R, Ragavendran A, Brand H, Erdin S, Cowan CA, Talkowski ME, Musunuru K. 2014. Low incidence of offtarget mutations in individual CRISPR-Cas9 and TALEN targeted human stem cell clones detected by whole-genome sequencing. Cell Stem Cell 15: 27-30.

Wang H, Yang H, Shivalila CS, Dawlaty MM, Cheng AW, Zhang F, Jaenisch R. 2013. One-step generation of mice carrying mutations in multiple genes by CRISPR/Cas-mediated genome engineering. Cell 153: 910918.

Wu X, Scott DA, Kriz AJ, Chiu AC, Hsu PD, Dadon DB, Cheng AW, Trevino AE, Konermann S, Chen S, et al. 2014. Genome-wide binding of the CRISPR endonuclease Cas9 in mammalian cells. Nat Biotechnol 32: 670-676.

Xie F, Ye L, Chang JC, Beyer AI, Wang J, Muench MO, Kan YW. 2014. Seamless gene correction of $\beta$-thalassemia mutations in patient-specific iPSCs using CRISPR/Cas9 and piggyBac. Genome Res 24: 1526-1533.

Yang L, Guell M, Byrne S, Yang JL, De Los Angeles A, Mali P, Aach J, KimKiselak C, Briggs AW, Rios X, et al. 2013. Optimization of scarless human stem cell genome editing. Nucleic Acids Res 41: 9049-9061.

Yang L, Grishin D, Wang G, Aach J, Zhang CZ, Chari R, Homsy J, Cai X, Zhao Y, Fan JB, et al. 2014. Targeted and genome-wide sequencing reveal single nucleotide variations impacting specificity of Cas9 in human stem cells. Nat Commun 5: 5507.

Zou J, Maeder ML, Mali P, Pruett-Miller SM, Thibodeau-Beganny S, Chou BK, Chen G, Ye Z, Park IH, Daley GQ, et al. 2009. Gene targeting of a disease-related gene in human induced pluripotent stem and embryonic stem cells. Cell Stem Cell 5: 97-110. 


\section{Genome Editing in Human Pluripotent Stem Cells}

Cory Smith, Zhaohui Ye and Linzhao Cheng

Cold Spring Harb Protoc; doi: 10.1101/pdb.top086819

\begin{tabular}{cc}
$\begin{array}{c}\text { Email Alerting } \\
\text { Service }\end{array}$ & Receive free email alerts when new articles cite this article - click here. \\
\hline $\begin{array}{c}\text { Subject } \\
\text { Categories }\end{array}$ & Browse articles on similar topics from Cold Spring Harbor Protocols. \\
& Embryonic Stem Cells (55 articles) \\
& Molecular Biology, general (1293 articles) \\
& Mutagenesis (57 articles) \\
& RNA (317 articles) \\
& RNA, general (269 articles) \\
& Stem Cells (46 articles) \\
& Stem Cells, general (70 articles) \\
& Transgenic Technology, general (188 articles) \\
\hline
\end{tabular}

